\title{
A Tighter Bhattacharyya Bound for Decoding Error Probability
}

Miguel Griot Student Member, IEEE, Wen-Yen Weng Student Member, IEEE, and Richard D. Wesel Senior Member, IEEE.

\begin{abstract}
The Bhattacharyya bound has been widely used to upper bound the pair-wise probability of error when transmitting over a noisy channel. However, the bound as it appears in most textbooks on channel coding can be improved by a factor of $1 / 2$ when applied to the frame error probability. For the particular case of symmetric channels, the pairwise error probability can also be improved by a factor of $1 / 2$. This letter provides a simple proof of these tighter bounds that has the same simplicity as the proof of the standard Bhattacharyya bound currently found in textbooks.
\end{abstract}

\section{Keywords}

Channel coding, Bhattacharyya bound, error probability.

\section{INTRODUCTION}

DEE Bhattacharyya bound has been widely used to upper bound the pair-wise prob-
ability of error when transmitting over a noisy channel. The bound as it appears in most textbooks on channel coding[1][2][3], is expressed as follows:

$$
P(X \rightarrow \tilde{X}) \leq \sum_{Y \in \mathcal{Y}} \sqrt{p(Y \mid \tilde{X}) p(Y \mid X)}
$$

where $X$ is the transmitted codeword, $\tilde{X}$ is any other possible codeword, $P(X \rightarrow \tilde{X})$ denotes the pairwise probability of decoding $\tilde{X}$, given that $X$ was transmitted, $\mathcal{Y}$ is the set of all possible received words. However, the bound can be improved by a factor of $1 / 2$. To our knowledge, this improved bound has been shown by Kailath in [4] in 1967, for the case of signal detection under two hypotheses, using Kolmogorov variational distance in a proof that is distinct (and more complex) than what is presented in this paper. His result was used

This work was supported by the Defence Advanced Research Project Agency under SPAWAR Systems Center San Diego Grant N66001-02-1-8938.

M. Griot, A. I. Vila Casado, W.-Y. Weng, H. Chan, and R. D. Wesel are with the Electrical Engineering Department, University of California, Los Angeles, CA 90095 USA (e-mail:[mgriot,wenyen,wesel]@ee.ucla.edu). 
in [5], but has remained unmentioned by modern textbooks ever since, and has never been applied to the probability of error for channel codes.

This letter shows that the Bhattacharyya bound for the frame error rate can be improved by a factor of $1 / 2$. We also show that in the case of symmetric channels, the bound for the pairwise error probability can also be improved by a factor of $1 / 2$. In Section II the tighter bounds for discrete channels are introduced and proofs for them are provided. For simplicity the statements and proofs shown in Section II are for discrete channels. However, these bounds and their proofs can be generalized easily for channels with real outputs. Section III delivers the conclusions.

\section{Improved BhatTACHARYyA BOUnds FOR DISCRETE CHANNELS}

We begin with a Lemma that suggests the looseness of (1) by including an additional term on the left hand-side. Denote the set of possible codewords of a certain code $C$ as $\mathcal{X}$.

Lemma II.1: Given any two codewords $X \in \mathcal{X}$ and $\tilde{X} \in \mathcal{X}$, then:

$$
P(X \rightarrow \tilde{X})+P(\tilde{X} \rightarrow X) \leq \sum_{Y \in \mathcal{Y}} \sqrt{p(Y \mid \tilde{X}) p(Y \mid X)}
$$

where $p(Y \mid X)$ is the probability of receiving $Y$ given that $X$ was transmitted.

Proof of Lemma II.1:

Given two possible codewords $X$ and $\tilde{X}$, assume without loss of generality that when $p(Y \mid X)=p(Y \mid \tilde{X})$, then $X$ is chosen by the decoder between the two. Using the union bound over both pairwise error probabilities, we get the following inequality:

$$
\begin{gathered}
P(X \rightarrow \tilde{X})+P(\tilde{X} \rightarrow X) \leq \\
\leq \sum_{Y \in \mathcal{Y}}[I(p(Y \mid \tilde{X})>p(Y \mid X)) p(Y \mid X)+I(p(Y \mid X) \geq p(Y \mid \tilde{X})) p(Y \mid \tilde{X})]
\end{gathered}
$$

where $I(\cdot)$ is the indicator function. Notice that when one of the indicator functions on (3) 
is 1 , the other indicator function is 0 . Therefore,

$$
P(X \rightarrow \tilde{X})+P(\tilde{X} \rightarrow X) \leq \sum_{Y \in \mathcal{Y}} \min (p(Y \mid \tilde{X}), p(Y \mid \tilde{X}))
$$

Now,

$$
\min (p(Y \mid \tilde{X}), p(Y \mid \tilde{X})) \leq \sqrt{p(Y \mid \tilde{X}) p(Y \mid X)}
$$

Applying the inequality in (5) to (4), we get (2), which proves the Lemma.

We will use Lemma II.1 to find the bound on the frame error probability, defined as:

$$
P_{e}=\sum_{X \in \mathcal{X}} P(\text { error } \mid X \text { transmitted }) P(X \text { transmitted })
$$

Theorem II.2: Given any discrete channel, and any code $\mathcal{X}$ with equal a-priori probabilities, i.e. $P(X)=P(\tilde{X})=1 /|\mathcal{X}|, \forall \mathcal{X}, \tilde{\mathcal{X}} \in \mathcal{X}$, the frame error probability can be upper bounded by:

$$
P_{e} \leq \frac{1}{2|\mathcal{X}|}\left(\sum_{X \in \mathcal{X}} \sum_{\tilde{X} \neq X} \sum_{Y \in \mathcal{Y}} \sqrt{p(Y \mid \tilde{X}) p(Y \mid X)}\right)
$$

\section{Proof of Theorem II.2}

Using the union bound of the pairwise error probabilities, the frame error probability is bounded by:

$$
P_{e} \leq \sum_{X \in \mathcal{X}} \sum_{\tilde{X} \neq X} P(X \rightarrow \tilde{X}) P(X)
$$

Since $P(X)=P(\tilde{X}), \forall X, \tilde{X} \in \mathcal{X},(8)$ can be rewritten as:

$$
P_{e} \leq \frac{1}{\mathcal{X}} \sum_{X \in \mathcal{X}} \sum_{\tilde{X} \neq X} P(X \rightarrow \tilde{X})
$$




$$
=\frac{1}{2}\left(\sum_{X \in \mathcal{X}} \sum_{\tilde{X} \neq X}(P(X \rightarrow \tilde{X})+P(\tilde{X} \rightarrow X)) \frac{1}{\mathcal{X}}\right),
$$

where (10) includes every term in the sum twice and divides by two. Applying Lemma II.1 to (10) we obtain (7), which proves the theorem.

Theorem II.3: For symmetric channels, the pairwise error probability $P(X \rightarrow \tilde{X})$ can be upper bounded by:

$$
P(X \rightarrow \tilde{X}) \leq \frac{1}{2} \sum_{Y \in \mathcal{Y}} \sqrt{p(Y \mid \tilde{X}) p(Y \mid X)} .
$$

Proof of Theorem II.3

For symmetric channels,

$$
P(X \rightarrow \tilde{X})=P(\tilde{X} \rightarrow X)=\frac{1}{2}(P(X \rightarrow \tilde{X})+P(\tilde{X} \rightarrow X)) .
$$

The theorem is proved by applying Lemma II.1 to the right-hand side of Eq. (12).

Note that for simplicity, we have stated and shown the bounds in the discrete domain. However, all the results shown in this section can be easily generalized to channels with real outputs, changing the $\sum_{Y \in \mathcal{Y}}$ to $\int_{Y \in \mathcal{Y}}$ and PMFs to the corresponding PDFs.

\section{Conclusions}

We have shown that the Bhattacharyya bound on the frame error probability as shown in textbooks on channel coding, can be made tighter simply multiplying the bound by a factor of $1 / 2$. We have also shown that this factor of $1 / 2$ can be applied in the pairwise error

probability for symmetric channels. For simplicity, proofs have been provided for discrete channels but they can easily be generalized to memoryless channels with real outputs.

\section{REFERENCES}

[1] Stephen B. Wicker. Error Control Systems for Digital Communication and Storage, chapter 12.3. Prentice Hall, Inc., 1995. 
[2] Stephen G. Wilson. Digital Modulation and Coding, chapter 4. Prentice Hall, Inc., 1996.

[3] Rolf Johannesson and Kamil Sh. Zigangirov. Fundamentals of Convolutional Coding, chapter 4. IEEE Press, 1999.

[4] Thomas Kailath. The divergence and Bhattacharyya distance measures in signal selection. IEEE Trans. on Comm. Technology, com-15, Feb. 1967.

[5] Martin E. Hellman and Josef Raviv. Probability of error, equivocation, and the Chernoff bound. IEEE Trans. on Info. Theory, 16, July 1970. 